\title{
Development of a disease specific questionnaire to measure health related quality of life in patients with chronic liver disease
}

\author{
Z M Younossi, G Guyatt, M Kiwi, N Boparai, D King
}

\begin{abstract}
Background and aims-To develop and assess a disease specific instrument for measuring health related quality of life (HRQL) in patients with chronic liver disease (CLD).

Methods-Based on responses from 60 patients with chronic liver disease, from 20 liver experts, and from a Medline search of the literature, items potentially affecting the HRQL of these patients were identified. A separate sample of 75 patients identified which items they found problematic and rated their importance. Results were explored using factor analysis; domains were chosen and items placed within domains. Redundant questions were eliminated and the final questionnaire was pretested in 10 patients. Using this instrument, HRQL was assessed in a further 133 patients with various types and stages of liver disease.

Results-Patients, experts, and the literature search identified 156 items of potential importance. Of these, 35 proved important to over $50 \%$ of 75 respondents in the item reduction sample. The factor analysis suggested six domains. After eliminating redundancies, the Chronic Liver Disease Questionnaire (CLDQ) included 29 items in the following domains: fatigue, activity, emotional function, abdominal symptoms, systemic symptoms, and worry. In pretesting, patients found the CLDQ clear and easy to complete in $\mathbf{1 0}$ minutes. In another 133 patients, the CLDQ showed a gradient between patients without cirrhosis, Child's A cirrhosis, and those with Child's B or C cirrhosis. CLDQ has evidence for moderate reliability at six months and seems to be responsive.
\end{abstract}

Conclusion-The CLDQ is short, easy to administer, produces both a summary score and domain scores, and correlates with the severity of liver disease.

(Gut 1999;45:295-300)

Keywords: quality of life; liver disease; liver specific quality of life; well being

The traditional "biomedical model" of health which is based on the basic sciences (molecular biology, genetics, physiology, biochemistry, etc.) is being integrated with the "social science model" of health, based on a psychosocial and economic foundation. This integrated ap- proach to clinical practice and research in medicine requires not only monitoring the traditional physiological and biochemical outcomes but also health related quality of life (HRQL). ${ }^{1-3}$

Chronic liver disease (CLD) results from a variety of disorders and is a major cause of morbidity and mortality worldwide. Death from liver disease or its complications is the ninth leading cause of mortality in the United States. About 5 million Americans suffer chronic infection from hepatitis $\mathrm{B}$ or $\mathrm{C}$ viruses (HBV or HCV) and millions more bear the consequences of other chronic hepatocellular or cholestatic liver disorders. ${ }^{4}$ Patients with chronic liver disease experience a variety of symptoms with profound negative impact on their HRQL. A systematic assessment of determinants of this impairment is currently not available. In a recent study of $\mathrm{HCV}$ infected individuals without cirrhosis, impairment in HRQL was not related to the degree of liver inflammation or mode of acquisition. ${ }^{5}$ The biology of chronic liver disease, and our clinical experience with these patients, is such that we would anticipate patients experiencing progressive symptoms and functional limitation with increasing severity of liver disease. Complications of cirrhosis (hepatic encephalopathy, ascites, spontaneous bacterial peritonitis, and recurrent variceal haemorrhages) can have further negative impact on patients' well being. On the other hand, the relation between HRQL impairment and severity of disease is unlikely to be very strong.

Many interventions in patients with CLD are geared primarily to make patients feel better and improve their HRQL. While investigators may choose from a variety of validated generic instruments for measurement of HRQL, these questionnaires may fail to detect small but important improvements in HRQL in patients with CLD. A liver specific instrument is likely to be more responsive to changes in HRQL that, while small, are nevertheless important..$^{6-13}$ Although preliminary data on modular instruments (based mainly on generic instruments) for liver disease are available, a disease specific instrument for patients with CLD is not available. In this article we describe the development of a disease specific HRQL

Abbreviations used in this paper: CLD, chronic liver disease; CLDQ, Chronic Liver Disease

Questionnaire; GRC, global rating of change; HBV, hepatitis B virus; HCV, hepatitis C virus; HRQL, health related quality of life; PBC, primary biliary cirrhosis; PSC, primary sclerosing cholangitis. 
instrument for CLD, the Chronic Liver Disease Questionnaire (CLDQ).

\section{Methods}

INSTRUMENT DEVELOPMENT PHASE

We have developed an evaluative instrument to measure longitudinal change over time within individuals with CLD. ${ }^{14-27}$ We deemed the following criteria essential for the final questionnaire.

(1) Both physical and emotional health should be measured.

(2) Items must reflect areas of function that are important to patients with CLD.

(3) The instrument must produce summary scores amenable to statistical analysis.

(4) The questionnaire should be responsive to clinically important changes, even if those changes are small.

(5) There should be evidence for its reliability and validity.

(6) Consideration of cost and efficiency dictate that the questionnaire be relatively short, between 20 and 30 items.

Patients

We selected patients from the hepatology practice of the Cleveland Clinic Foundation. We included patients if they had an established diagnosis of CLD, were older than 18 years of age, and lacked any other chronic disorders that may affect the HRQL. We also excluded patients if they had psychiatric or emotional problems, or language or cognitive difficulties that prevented reliable completion of the questionnaire. There was no overlap in the patients for each phase of development and testing.

This project was reviewed and fully approved by the Investigational Review Board of the Cleveland Clinic Foundation.

Item selection

We began by constructing a list of items likely to be important to patients with CLD. Sources of items included previous studies examining the impact of CLD on patients' lives ${ }^{16-24}$ and generic measures of HRQL. ${ }^{11-13}$ Additionally, 20 hepatologists and experts with extensive experience in the care of patients with liver disease completed an open ended questionnaire in which they listed items that have a significant impact on quality of life of patients with CLD; the importance of each item was ranked on a five point scale where 1 was least important and 5 was most important. Interviews with 60 patients with CLD were carried out (two focus groups of eight patients each, telephone interviews of 10 patients, and the remainder face to face individual interviews) which supplemented the list of items for the initial questionnaire.

Item reduction

Three principles guided our approach to item reduction. Firstly, our primary criterion for including an item was the impact of the item on the population (how often patients labelled the item as a problem for them, and the importance they attached to it). Secondly, in order to decrease variability of response and reduce any impact of idiosyncratic response to a given question (that is, helping to ensure validity and responsiveness), each domain must include three items. Thirdly, we used factor analysis as a strategy to help place items in different domains.

We interviewed 75 patients for the item reduction phase. Patients identified items that were problems for them. For those items that patients labelled as a problem, they rated the importance on a five point scale where 1 was least important and 5 was most important.

For each item, we calculated an "impact score", the product of the proportion of patients identifying the item as a problem (frequency), and the mean importance attributed to that item. The impact score for each item could range from 0 to 5 .

We conducted a factor analysis of items endorsed as problematic by more than $50 \%$ of the patients. Factor analysis was performed to guide placement of items into different domains. We combined this with judgement to make sure that placement of an item into a domain made intuitive sense. A principle component analysis identified items with an eigenvalue of greater than 1 . The eigenvalue rises as the proportion of variance explained by a factor increases, and investigators often use a cut of 1 to identify factors that warrant further consideration. We also used a scree plot to help decide on possible numbers of domains. The scree plot presents the eigenvalues of each factor in descending order, and helps determine where there is a rapid drop in the proportion of variance explained. In this case, the scree plot suggested a model of up to six factors. We then repeated the factor analysis using a varimax rotation, examining four, five, and six factor solutions, and chose the solution that made the most intuitive sense. The varimax rotation facilitates separation of factors with an optimal balance of variance explained by each factor.

\section{Questionnaire construction and pretesting}

We constructed appropriate questions for each item identified for the questionnaire, and specified response options using seven point scales, ranging from the worst (1) to the best (7) possible function. Previous work suggests that seven point scales combine excellent responsiveness with ease of administration and patient understanding. ${ }^{28}{ }^{29}$ Ten patients completed the self administered CLDQ, and commented on each question. We made necessary changes in the questionnaire construction and wording based on their comments.

ASSESSMENT OF HRQL USING CLDQ

Cross sectional administration of CLDQ

We administered the final CLDQ and MOSShort Form 36 (SF-36) to patients with a variety of types and stages of liver disease, recording clinical and demographic data at the time of questionnaire administration. We chose our overall sample size target of 130, hoping that we would recruit approximately 30 patients in each category of disease, allowing us to detect differences between groups of 0.6 standard deviation units. We used analysis of variance 
(ANOVA) to compare scores of patients with different stages of liver disease. We classified patients as non-cirrhotics, early cirrhotics (Child's A), and advanced cirrhotics (Child's B and C) using modified Child-Pugh classification. ${ }^{30}$ We considered a $\mathrm{p}$ value of less than 0.05 to be significant.

\section{Longitudinal administration of CLDQ}

We administered CLDQ and SF-36 to patients six months after the original administration. Global rating of change (GRC) was also administered to determine whether patients were stable or had changed. To assess reliability, intraclass correlation coefficients were calculated according to Shrout and Fleiss. ${ }^{31}$ Pearson correlation was used to see whether changes in CLDQ scores (or its domains) or SF-36 scores, correlated with clinical change as determined by GRC.

\section{Results}

INSTRUMENT DEVELOPMENT PHASE

Item selection

We interviewed 60 patients of whom $61 \%$ were women. Their age was 51 (10) years. Of these patients, 20 had hepatocellular liver disease (Child's $\leqslant \mathrm{A} 7$; seven, chronic hepatitis C (CAH-C); four, chronic hepatitis B (CAH-B); nine, other types of liver disease), 20 cholestatic liver disease (Child's $\leqslant \mathrm{A} ; 14$, primary biliary cirrhosis; six, primary sclerosing cholangitis), and 20 advanced liver disease of both types
(Child's $\geqslant B 8$; seven, viral hepatitis B and C related cirrhosis; five, alcohol related cirrhosis; four, primary biliary cirrhosis; two, primary sclerosing cholangitis; two, other types of liver disease). We identified 156 items that may impact on the HRQL of patients with CLD. We included these items in our item reduction questionnaire.

\section{Patients for item reduction}

We administered the item reduction questionnaire to 75 patients, $53.3 \%$ of whom were women. Their age was 51 (8) years, and $36 \%$ of patients had cholestatic liver diseases (16, primary biliary cirrhosis (PBC); 11, primary sclerosing cholangitis (PSC)) while 48 (64\%) patients had hepatocellular liver disease (15, CAH-C; four CAH-B; nine, alcohol related liver disease (ALD); 20, other types of liver disease). Twenty two (29.3\%) had no cirrhosis, 29 $(38.7 \%)$ had early cirrhosis (Child's $\leqslant \mathrm{A} 7$ ), and 24 patients $(32 \%)$ had advanced cirrhosis (Child's $\geqslant B 8$ ). Table 1 presents the items that over $50 \%$ of the patients identified as a problem.

\section{Item reduction and factor analysis}

Thirty five items were identified as important by over $50 \%$ of the respondents (table 1 ). A principle component analysis and scree plot suggested an up to six factor model. Factor analysis was performed; it assigned each item into one of the six factors (table 1).

Table 1 Impact scores and factor loadings of the items from the item reduction step

\begin{tabular}{|c|c|c|c|c|}
\hline CLDQ items & $\begin{array}{l}\text { Proportion } \\
\text { with problem }\end{array}$ & Importance & Impact & $\begin{array}{l}\text { Factor } \\
\text { loadings }\end{array}$ \\
\hline \multicolumn{5}{|l|}{ Factor 1} \\
\hline Decreased strength & 0.69 & 3.95 & 2.73 & 0.69 \\
\hline Fatigue & 0.80 & 3.88 & 3.10 & 0.82 \\
\hline Decreased level of energy & 0.81 & 4.04 & 3.27 & 0.79 \\
\hline Lack of energy & 0.59 & 4.24 & 2.50 & 0.75 \\
\hline Feeling weak & 0.63 & 3.88 & 2.44 & 0.83 \\
\hline Feeling worn out & 0.70 & 3.89 & 2.72 & 0.79 \\
\hline Feeling sleepy during the day & 0.80 & 3.55 & 2.84 & 0.53 \\
\hline Tired in the morning when you wake up & 0.59 & 4.03 & 2.38 & 0.57 \\
\hline Tired in the afternoon & 0.77 & 3.37 & 2.59 & 0.58 \\
\hline Drowsiness & 0.63 & 3.55 & 2.24 & 0.61 \\
\hline Problem carrying heavy objects & 0.55 & 3.49 & 1.92 & 0.55 \\
\hline Anxiety & 0.69 & 3.73 & 2.57 & 0.54 \\
\hline \multicolumn{5}{|l|}{ Factor 2} \\
\hline Feeling depressed & 0.56 & 4.00 & 2.24 & 0.51 \\
\hline Irritability & 0.55 & 4.03 & 2.22 & 0.48 \\
\hline Mood swings & 0.52 & 4.09 & 2.13 & 0.51 \\
\hline Difficulty sleeping at night & 0.61 & 3.77 & 2.30 & 0.71 \\
\hline Unable to fall asleep at night & 0.58 & 3.73 & 2.16 & 0.69 \\
\hline The need to get up at night to urinate & 0.63 & 2.98 & 1.88 & 0.40 \\
\hline Not feeling rested in the morning & 0.67 & 3.84 & 2.57 & 0.61 \\
\hline \multicolumn{5}{|l|}{ Factor 3} \\
\hline Worried about the impact your disease has on your family & 0.64 & 3.90 & 2.50 & 0.74 \\
\hline Worried your symptoms will develop into a major problem & 0.73 & 4.02 & 2.93 & 0.79 \\
\hline Worried never feeling any better & 0.52 & 4.36 & 2.27 & 0.60 \\
\hline Worried condition getting worse & 0.83 & 4.08 & 3.39 & 0.81 \\
\hline \multicolumn{5}{|l|}{ Factor 4} \\
\hline Feeling bloated & 0.66 & 3.48 & 2.30 & 0.80 \\
\hline Abdominal discomfort & 0.55 & 3.43 & 1.89 & 0.66 \\
\hline Abdominal bloating & 0.58 & 3.81 & 2.21 & 0.83 \\
\hline Muscle cramps at night & 0.56 & 3.58 & 2.00 & 0.47 \\
\hline \multicolumn{5}{|l|}{ Factor 5} \\
\hline Trouble lifting heavy objects & 0.53 & 3.59 & 1.90 & 0.51 \\
\hline Change in eating habits & 0.56 & 3.44 & 1.93 & 0.76 \\
\hline Not able to eat as much as before & 0.56 & 2.81 & 1.57 & 0.58 \\
\hline Napping during the day & 0.61 & 3.23 & 1.97 & 0.56 \\
\hline \multicolumn{5}{|l|}{ Factor 6} \\
\hline Shortness of breath & 0.53 & 3.88 & 2.06 & 0.49 \\
\hline Dry mouth & 0.61 & 3.33 & 2.03 & 0.52 \\
\hline Worried about the availability of a liver if you need a transplant & 0.55 & 4.60 & 2.53 & 0.64 \\
\hline Itching & 0.53 & 3.24 & 1.82 & 0.40 \\
\hline
\end{tabular}

Proportion of variance explained: factor $1,0.224$; factor $2,0.122$; factor $3,0.110$; factor $4,0.099$; factor 5, 0.098; factor 6, 0.056 . 
Table 2 CLDQ scores according to severity of liver disease

\begin{tabular}{llllll}
\hline \multicolumn{5}{l}{ Stage of liver disease } \\
\cline { 2 - 6 } Domain (score 1-7) & $\begin{array}{l}\text { No cirrhosis } \\
(n=45)\end{array}$ & $\begin{array}{l}\text { Child's } A \\
(n=43)\end{array}$ & $\begin{array}{l}\text { Child's } B \\
(n=31)\end{array}$ & $\begin{array}{l}\text { Child's } C \\
(n=14)\end{array}$ & $p$ Value \\
\hline Abdominal symptoms (AB) & $5.50(1.58)$ & $4.88(1.75)$ & $4.31(1.69)$ & $3.60(2.00)$ & 0.001 \\
Fatigue (FA) & $4.48(1.59)$ & $3.57(1.56)$ & $2.74(1.13)$ & $3.13(1.59)$ & $<0.001$ \\
Systemic symptoms (SY) & $5.36(1.35)$ & $4.74(1.24)$ & $4.03(1.29)$ & $4.23(1.42)$ & $<0.001$ \\
Activity (AC) & $5.64(1.31)$ & $4.57(1.62)$ & $3.40(1.43)$ & $3.21(1.32)$ & $<0.001$ \\
Emotional function (EM) & $4.67(1.05)$ & $4.50(0.95)$ & $4.01(1.15)$ & $4.04(0.75)$ & 0.020 \\
Worry (WO) & $5.19(1.32)$ & $4.57(1.55)$ & $4.10(1.60)$ & $3.83(1.70)$ & 0.004 \\
CLDQ overall score & $5.14(1.14)$ & $4.47(1.12)$ & $3.76(1.09)$ & $3.76(1.19)$ & $<0.001$ \\
\hline
\end{tabular}

Table 3 SF-36 scores according to severity of liver disease

\begin{tabular}{lllllr}
\hline \multicolumn{7}{l}{ Stage of liver disease } \\
\cline { 2 - 6 } Domain & $\begin{array}{l}\text { No cirrhosis } \\
(n=45)\end{array}$ & $\begin{array}{l}\text { Child's } A \\
(n=43)\end{array}$ & $\begin{array}{l}\text { Child's } B \\
(n=31)\end{array}$ & $\begin{array}{l}\text { Child's } C \\
(n=14)\end{array}$ & $p$ Value \\
\hline Physical functioning (PF) & $79.57(25.7)$ & $57.95(26.0)$ & $36.21(26.5)$ & $31.89(24.2)$ & $<0.001$ \\
Role-physical (RP) & $65.79(44.4)$ & $43.42(41.0)$ & $23.28(36.6)$ & $5.36(14.47)$ & $<0.001$ \\
Bodily pain (BP) & $72.51(26.5)$ & $57.59(26.2)$ & $46.55(27.1)$ & $54.38(31.9)$ & 0.002 \\
General health (GH) & $63.65(21.7)$ & $45.42(19.6)$ & $35.46(21.1)$ & $30.33(23.3)$ & $<0.001$ \\
Vitality (VT) & $49.91(11.2)$ & $45.51(8.57)$ & $48.15(8.7)$ & $50.77(7.32)$ & 0.150 \\
Social functioning (SF) & $76.6(24.5)$ & $67.95(26.7)$ & $52.59(30.3)$ & $44.23(30.0)$ & $<0.001$ \\
Role-emotion (RE) & $73.68(41.9)$ & $65.77(41.9)$ & $40.48(44.8)$ & $30.77(39.6)$ & 0.001 \\
Mental health (MH) & $48.1(14.7)$ & $60.21(16.3)$ & $62.81(14.3)$ & $57.54(16.9)$ & $<0.001$ \\
Mental component summary score & $40.99(7.1)$ & $45.8(5.8)$ & $44.15(6.1)$ & $49.2(5.6)$ & 0.013 \\
Physical component summary score & $49.82(13.6)$ & $36.96(11.4)$ & $39.1(11.6)$ & $27.8(9.1)$ & $<0.001$ \\
\hline
\end{tabular}

Pretesting of questionnaire

We began by pretesting the 35 item questionnaire. In pretesting, patients felt that there was excessive redundancy in seven of the items in the fatigue domain. They were "fatigue", "feeling weak", "lack of energy", "tired in the afternoon", "tired in the morning", "decreased level of energy", and "feeling worn out". These items were combined into two items. Feeling worn out, feeling weak, and fatigue were combined as one item and decreased level of energy, lack of energy, and tired in the morning and afternoon as a separate item. The activity domain also had redundancies: "problem carrying heavy objects" and "problem lifting heavy objects" were combined as one item. After eliminating redundancies, 29 items remained which were distributed in six domains. The items were presented as closed ended questions with seven point scale response options. In general, patients found the presentation of the questionnaire clear and satisfactory, and they utilised the full range of response options. On average, it take about 10 minutes to complete the questionnaire. The CLDQ is summarised in the Appendix.

ASSESSMENT OF HRQL USING CLDQ

Cross sectional administration of CLDQ

Of the 133 patients with CLD who completed the CLDQ in the next phase of the study, $47 \%$ were women and their average age was 50 (11) years. Of these patients, $30(23 \%)$ patients had cholestatic liver diseases (PBC, PSC) while $103(77 \%)$ patients had hepatocellular liver disease. Forty five (34\%) had no cirrhosis, 43 $(32 \%)$ had Child's A, 31 (23\%) Child's B, and $14(11 \%)$ had Child's $C$ cirrhosis.

The overall CLDQ score for patients with no cirrhosis (5.14 (1.14)) was higher than for those with early cirrhosis or Child's A (4.47 (1.12)) and those with advanced cirrhosis or Child's B and C (3.76 (1.09) and 3.76 (1.19), respectively) ( $\mathrm{p}<0.001$, ANOVA). We observed a similar gradient across each of the six domains (table 2). Similar reductions in scores were seen for SF-36, except for vitality (VT) and mental health $(\mathrm{MH})$ scales. As severity of liver disease worsened, the physical component summary score (PCS) of SF-36 performed better than the mental component summary score (MCS) (table 3).

\section{Longitudinal administration of CLDQ}

Of the 46 patients with CLD who completed the CLDQ a second time (six months after the initial administration), $50 \%$ were women and their average age was 53 (11) years. Of these patients, 15 patients (33\%) had cholestatic liver diseases (PBC, PSC), while 31 (77\%) patients had hepatocellular liver disease. Sixteen patients (35\%) had no cirrhosis, $16(35 \%)$ had Child's A, 12 (26\%) Child's B, and two (4\%) had Child's C cirrhosis.

Of these patients, $15(33 \%)$ had no change in their global ratings of change scores and were considered stable. The intraclass correlations (ICC) for each domain were as follows: fatigue $(\mathrm{ICC}=0.72)$, emotional function $(\mathrm{ICC}=0.68)$, abdominal symptoms $(\mathrm{ICC}=0.63)$, worry $(\mathrm{ICC}=0.58)$, activity (ICC=0.43), systemic symptoms $(\mathrm{ICC}=0.23)$, and overall CLDQ $(\mathrm{ICC}=0.59)$.

Of patients that deteriorated, changes in CLDQ scores over the six month period (change in CLDQ score or change in domain scores) were correlated with GRC scores. Changes in the overall CLDQ score correlated highly with GRC $(r=0.84, \mathrm{p}=0.02)$. The fatigue domain of CLDQ and the abdominal symptom domain of CLDQ, both correlated highly with GRC ( $r=0.83$ and $r=0.90$, respectively; $\mathrm{p}=0.006$ ). In this cohort of patients, there were not sufficient changes in the remainder of domains to reach statistical significance. In the same cohort of patients that 
deteriorated, changes in SF-36 scores were correlated with changes in GRC. This correlation was 0.23 for MCS and 0.23 for PCS $(\mathrm{p}=0.57)$.

\section{Discussion}

We have developed the first disease specific HRQL instrument for patients with CLD (Chronic Liver Disease Questionnaire) using a comprehensive methodological framework ${ }^{6}$ established for development of other disease specific, HRQL instruments. ${ }^{6-10}$ Our approach to item selection which utilised a variety of sources including input from patients with diverse types and stages of liver disease was comprehensive and ensured that we captured all important items. Involvement of 75 patients (again with a variety of types and stages of liver disease) in the item reduction process further enhances the content validity of our questionnaire. Designation of different items into appropriate domains through factor analysis ensures that the domain scores represent specific aspects of HRQL. Finally, our pretesting provides evidence that the CLDQ is clear and that items are easy to understand.

We recommend that, by dividing each domain score by the number of items in the domain, investigators present CLDQ results on a 1 to 7 scale. A consistent presentation of results on a seven point scale facilitates their interpretability. This is the case because, for a number of similarly structured disease specific HRQL measures, a change of 0.5 on the 1 to 7 scale approximates the important difference in questionnaire score..$^{25-29}$ While empirical demonstration would strengthen our inference that the same interpretation applies to CLDQ, the repeated finding with different questionnaires and different measurement techniques suggests that this may well be the case.

We have administered CLDQ to a cohort of patients with a variety of types and stages of CLD. Our data show that as liver disease becomes more severe, patients' HRQL as measured by the CLDQ deteriorates. This supports the construct validity of the instrument as a cross sectional measure of HRQL for CLD. Although scale scores for SF-36 also deteriorate with disease severity, this was not true for all scales. Specifically the vitality and mental health scales of SF-36 did not capture this change in severity of liver disease.

Patients with advanced cirrhosis (Child's B and C) showed similar HRQL scores measured both by generic and disease specific instruments. One possible explanation of this finding is that the clinical deterioration between Child's B and C is not accompanied by a corresponding deterioration in HRQL. Alternatively, neither the generic instrument nor the CLDQ may focus sufficiently on issues of particular concern to Child's C patients, and thus fail to discriminate the latter stages of liver failure. Finally, the small sample size of the Child's C group (14 patients) could explain our inability to show a difference between groups.

In further testing, CLDQ seems to have moderate reliability after six months of readministration. Given that CLDQ scores corre- lated highly with clinical evidence of deterioration (measured by GRC), this instrument seems to be more responsive than the generic SF-36.

Limitations of our study include our decision to focus on items that were important to over $50 \%$ of our population. Thus, it is possible we excluded items that are important to only a particular subpopulation. Our pragmatic goal of creating a widely applicable and efficient instrument suitable for clinical trials mandated this approach which carries with it this inevitable limitation.

Additional concerns include our recruiting patients from a single tertiary care medical centre. Although we have sampled patients from both spectrums of disease severity (non-cirrhotics to Child's C cirrhotics), a selection bias is still possible. However, given the nature of quality of life concerns, a radical difference across populations seems unlikely. Finally, we have not yet investigated the responsiveness of the CLDQ, its ability to detect important change over time even if that change is small. We are addressing these issues in ongoing studies of the CLDQ in nontertiary settings and efficacy trials of patients with a variety of types of liver disease.

In summary, CLDQ is the first disease specific HRQL instrument that has been systematically developed. Using this instrument and SF-36, HRQL in patients with chronic liver disease deteriorates as the disease becomes more severe. This impairment in HRQL seems better detected by the disease specific instrument: CLDQ. Supplementing other clinical end points, CLDQ can be useful in clinical research involving patients with chronic liver disease.

Results were partly presented at the 56th Annual Meeting of the American Association for the Study of Liver Disease (1997), Chicago, Illinois; and Digestive Disease Week (1998), New Orleans, Louisiana. This research was supported in part by a grant from IH Page Centre of the Cleveland Clinic Foundation, and American College of Gastroenterology, Junior Faculty Award to ZMY.

1 Bergner M. Quality of life, health status and clinical research. Medical Care 1989;27(suppl):S148-56.

2 Testa M, Simonson D. Assessment of quality of life outcomes. N Engl F Med 1996;13:835-40.

3 Wilson I, Cleary P. Linking clinical variables with healthrelated quality of life. $7 A M A$ 1995;273:59-65.

4 Everhart J. Digestive diseases in the United States: epidemiology and impact. NIH Publication No. 94-1447, 1994.

5 Foster G, Goldin R, Thomas H. Chronic hepatitis C virus infection causes a significant reduction in quality of life in the absence of cirrhosis. Hepatology 1998;27:209-12.

6 Guyatt G, Bombardier C, Tugwell P. Measuring disease specific quality of life in clinical trials. Can Med Assoc 7 1986;134:889-95.

7 Kongpatanakul S, Strom B. Quality of life, health status and clinical drug research. Pharmacoeconomics 1992;2:8-14.

8 Fowlie M, Berkeley J. Quality of life-a review of the literaFowlie M, Berkeley J. Quality of life--
ture. Family Practice 1987;4:226-34.

9 Guyatt G, Walter S, Norman G. Measuring change over time: assessing the usefulness of evaluative instrument. $\mathcal{F}$ Chron Dis 1987;40:171-8.

10 Guyatt G, Berman L, Townsend M, et al. A measure of quality of life for clinical trials in chronic lung disease. Tho$\operatorname{rax} 1987 ; 42: 773-80$.

11 Bruin A, De Witte L, Stevens F, et al. Sickness impact profile: the state of the art of a generic functional status measure. Soc Sci Med 1992;35:1003-10.

12 Bergnen M, Bobbitt R, Carter W, et al. The sickness impact profile: development and final revision of a health status measure. Medical Care 1981;19:787-805.

13 Ware J, Sherbourne C. The MOS 36-item Short Form Health Survey (SF-36). I. Conceptual framework and item selection. Medical Care 1992;30:473-84.

14 Kirshner B, Guyatt GH. A methodologic framework for assessing health indices. F Chron Dis 1985;38:27-36. 
15 Guyatt GH, Kirshner B, Jaeschke R. Measuring health status: what are the necessary measurement properties. $\mathcal{F}$

16 Tarter R, Switala J, Arria A, et al. Quality of life before and after orthotopic liver transplantation. Arch Intern Med 1991;151:1521-4

17 Levy M, Goldstein R, Husberg B, et al. Baylor update: outcome analysis in liver transplantation. In: Clinical transplants. Los Angeles: UCLA Tissue Typing Laboratory, 1993:161-73.

18 Kechtle S, Fleming M, Barry K, et al. Liver transplantation in alcoholics: assessment of psychological health and work activity. Transplant Proc 1993;25:1916-19.

19 Moore K, Burrows R, Jones M, et al. Control evaluation of cognitive functioning, mood state, and quality of life postliver transplant. Transplant Proc 1992:24:202-6.

20 Lundgren $M$, Kristiansson $M$, Ericzon B, et al. Improved quality of life after liver transplantation. Transplant Proc 1994;26:1779.

21 Haagsma E, Klompmaker I, Verwer R, et al. Long term results after liver transplantation in adults. Scand $\mathcal{F}$ Gastroresults after liver trans

22 Belle S, Porayko M, Hoofnagle J, et al. Changes in quality of life after liver transplantation among adults. Liver Transplantation and Surgery 1997;3:93-104.

23 Davis G, Balart L, Schiff E, et al. Assessing health-related quality of life in chronic hepatitis C using the sicknes impact profile. Clin Ther 1994;16:334-43.

24 Carithers R, Sugano D, Bayliss M. Health assessment for chronic HCV: results of quality of life. Dig Dis Sci 1996;41: $75 \mathrm{~S}-78 \mathrm{~S}$

25 Jaeschke R, Guyatt G, Keller J, et al. Measurement of health status: ascertaining the meaning of a change in quality-oflife questionnaire score. Controlled Clin Trials 1989:10:40715.

26 Juniper EF, Guyatt GH, William A, et al. Determining a minimal important change in a disease-specific quality of life questionnaire. $\mathcal{F}$ Clin Epidemiol 1994;47:81-7.

27 Juniper EF, Guyatt GH, Griffith LE, et al. Interpretation of Rhinoconjunctivitis Quality of Life Questionnaire data. I Rhinoconjunctivitis Quality of
Allergy Clin Immunol (in press)

28 Guyatt GH, Townsend M, Berman LB, et al. A comparison of Likert and visual analogue scales for measuring change in function. F Chron Dis 1987;40:1129-33.

29 Jaeschke R, Singer J, Guyatt GH. A comparison of seven point and visual analogue scales: data from a randomized trial. Controlled Clinical Trials 1990;11:43-51.

30 Pugh RN, Murray-Lyon IM, Dawson JL, et al. Transection of the oesophagus varices. Brf Surg 1973;60:646-9.

31 Shrout PE, Fleiss JL. Intraclass correlations: uses in assessing rater reliability. Psychiatr Bull 1979;86:420-8.

(C) 1999 Gut

\section{Appendix}

THE CHRONIC LIVER DISEASE QUESTIONNAIRE (CLDQ) - QUALITY OF LIFE INDEX FOR PATIENTS

WITH CHRONIC LIVER DISEASE

This questionnaire is designed to find out how you have been feeling during the last two weeks. You will be asked about your symptoms related to your liver disease, how you have been affected in doing activities, and how your mood has been. Please complete all of the questions and select only one response for each question.

RESPONSE OPTIONS

1 All of the time

2 Most of the time

3 A good bit of the time

4 Some of the time

5 A little of the time

6 Hardly any of the time

7 None of the time

QUESTIONS

1. How much of the time during the last two weeks have you been troubled by a feeling of abdominal bloating?

2. How much of the time have you been tired or fatigued during the last two weeks?

3. How much of the time during the last two weeks have you experienced bodily pain?
4. How often during the last two weeks have you felt sleepy during the day?

5. How much of the time during the last two weeks have you experienced abdominal pain?

6. How much of the time during the last two weeks has shortness of breath been a problem for you in your daily activities?

7. How much of the time during the last two weeks have you not been able to eat as much as you would like?

8. How much of the time in the last two weeks have you been bothered by having decreased strength?

9. How often during the last two weeks have you had trouble lifting or carrying heavy objects?

10. How often during the last two weeks have you felt anxious?

11. How often during the last two weeks have you felt a decreased level of energy?

12. How much of the time during the last two weeks have you felt unhappy?

13. How often during the last two weeks have you felt drowsy?

14. How much of the time during the last two weeks have you been bothered by a limitation of your diet?

15. How often during the last two weeks have you been irritable?

16. How much of the time during the last two weeks have you had difficulty sleeping at night?

17. How much of the time during the last two weeks have you been troubled by a feeling of abdominal discomfort?

18. How much of the time during the last two weeks have you been worried about the impact your liver disease has on your family?

19. How much of the time during the last two weeks have you had mood swings?

20. How much of the time during the last two weeks have you been unable to fall asleep at night?

21. How often during the last two weeks have you had muscle cramps?

22. How much of the time during the last two weeks have you been worried that your symptoms will develop into major problems?

23. How much of the time during the last two weeks have you had a dry mouth?

24. How much of the time during the last two weeks have you felt depressed?

25. How much of the time during the last two weeks have you been worried about your condition getting worse?

26. How much of the time during the last two weeks have you had problems concentrating?

27. How much of the time have you been troubled by itching during the last two weeks?

28. How much of the time during the last two weeks have you been worried about never feeling any better?

29. How much of the time during the last two weeks have you been concerned about the availability of a liver if you need a liver transplant?

\section{DOMAINS}

Abdominal symptoms (AS): Items 1, 5, 17

Fatigue (FA): Items 2, 4, 8, 11, 13

Systemic symptoms (SS): Items 3, 6, 21, 23, 27

Activity (AC): Items 7, 9, 14

Emotional function (EF): Items 10, 12, 15, 16, 19, 20, 24,26

Worry (WO): Items 18, 22, 25, 28, 29

(C) 1999 Younossi 